\title{
RECURSIVE PREDICATES AND QUANTIFIERS $\left({ }^{1}\right)$
}

\author{
BY \\ S. C. KLEENE
}

This paper contains a general theorem on the quantification of recursive predicates, with applications to the foundations of mathematics. The theorem (Theorem II) is a slight extension of previous results on Herbrand-Gödel general recursive functions $\left({ }^{2}\right)$, while the applications include theorems of Church (Theorem VII) ${ }^{3}$ ) and Gödel (Theorem VIII) $\left({ }^{4}\right)$ and other incompleteness theorems. It is thought that in this treatment the relationship of the results stands out more clearly than before.

The general theorem asserts that to each of an enumeration of predicate forms, there is a predicate not expressible in that form. The predicates considered belong to elementary number theory.

The possibility that this theorem may apply appears whenever it is proposed to find a necessary and sufficient condition of a certain kind for some given property of natural numbers; in other words, to find a predicate of a given kind equivalent to a given predicate. If the specifications on the predicate which is being sought amount to its having one of the forms listed in the theorem, then for some selection of the given property a necessary and sufficient condition of the desired kind cannot exist.

In particular, it is recognized that to find a complete algorithmic theory for a predicate $P(a)$ amounts to expressing the predicate as a recursive predicate. By one of the cases of the theorem, this is impossible for a certain $P(a)$, which gives us Church's theorem.

Again, when we recognize that to give a complete formal deductive theory (symbolic logic) for a predicate $P(a)$ amounts to finding an equivalent predicate of the form $(E x) R(a, x)$ where $R(a, x)$ is recursive, we have immediately Gödel's theorem, as another case of the general theorem.

Still another application is made, when we consider the nature of a constructive existence proof. It appears that there is a proposition provable classically for which no constructive proof is possible (Theorem $\mathrm{X}$ ).

The endeavor has been made to include a fairly complete exposition of definitions and results, including rèlevant portions of previous theory, so that

Presented to the Society, September 11, 1940; received by the editors February 13, 1942. In the abstract of this paper, Bull. Amer. Math. Soc. abstract 46-11-464, erratum: line 4, for "for." read "for all.".

(1) A part of the work reported in this paper was supported by the Institute for Advanced Study and the Alumni Research Foundation of the University of Wisconsin.

(2) Gödel $[2, \S 9]$ (see the bibliography at the end of the paper).

(3) Church [1].

(4) Gödel [1, Theorem VI]. 
the paper should be self-contained, although some details of proof are omitted.

The general theorem is obtained quickly in Part I from the properties of the $\mu$-operator, or what essentially was called the $p$-function in the author's dissertation $\left({ }^{5}\right)$. Part II contains some variations on the theme of Part I, and may be omitted by the cursory reader. The applications to foundational questions are in Part III, only a few passages of which depend on Part II.

\section{THE GENERAL THEOREM ON RECURSIVE PREDICATES AND QUANTIFIERS}

1. Primitive recursive functions. The discussion belongs to the context of the informal theory of the natural numbers

$$
0,1,2, \cdots, x, x^{\prime}, \cdots .
$$

The functions which concern us are number-theoretic functions, for which the arguments and values are natural numbers.

We consider the following schemata as operations for the definition of a function $\phi$ from given functions appearing in the right members of the equations ( $c$ is any constant natural number):

$$
\begin{aligned}
& \phi(x)=x^{\prime}, \\
& \phi\left(x_{1}, \cdots, x_{n}\right)=c \text {, } \\
& \phi\left(x_{1}, \cdots, x_{n}\right)=x_{i} \text {, } \\
& \phi\left(x_{1}, \cdots, x_{n}\right)=\theta\left(\chi_{1}\left(x_{1}, \cdots, x_{n}\right), \cdots, \chi_{m}\left(x_{1}, \cdots, x_{n}\right)\right) \text {, } \\
& \left\{\begin{array}{l}
\phi(0)=c \\
\phi\left(y^{\prime}\right)=\chi(y, \phi(y)),
\end{array}\right. \\
& \left\{\begin{aligned}
\phi\left(0, x_{1}, \cdots, x_{n}\right) & =\psi\left(x_{1}, \cdots, x_{n}\right) \\
\phi\left(y^{\prime}, x_{1}, \cdots, x_{n}\right) & =\chi\left(y, \phi\left(y, x_{1}, \cdots, x_{n}\right), x_{1}, \cdots, x_{n}\right) .
\end{aligned}\right.
\end{aligned}
$$

Schema (I) introduces the successor function, Schema (II) the constant functions, and Schema (III) the identity functions. Schema (IV) is the schema of definition by substitution, and Schema (V) the schema of primitive recursion. Together we may call them (and more generally, schemata reducible to a series of applications of them) the primitive recursive schemata.

A function $\phi$ which can be defined from given functions $\psi_{1}, \cdots, \psi_{k}$ by a series of applications of these schemata we call primitive recursive in the given functions; and in particular, a function $\phi$ definable ab initio by these means, primitive recursive.

Now let us consider number-theoretic predicates, that is, propositional functions of natural numbers.

(5) Kleene [1, \$18]. 
In asserting propositions, and in designating predicates, we use a logical symbolism, as follows. Operations of the propositional calculus: \& (and), $\vee$ (or), - (not), $\rightarrow$ (implies), $\equiv$ (equivalent). Quantifiers: $(x)$ (for all $x$ ), $(E x)$ (there exists an $x$ such that). These operations may be taken either in the sense of classical mathematics, or in the sense of constructive or intuitionistic mathematics, except where one or the other of the two interpretations is specified.

A predicate $P\left(x_{1}, \cdots, x_{n}\right)$ is said to be primitive recursive, if there is a primitive recursive function $\pi\left(x_{1}, \cdots, x_{n}\right)$ such that

$$
P\left(x_{1}, \cdots, x_{n}\right) \equiv \pi\left(x_{1}, \cdots, x_{n}\right)=0 .
$$

We can without loss of generality restrict $\pi$ to take only 0 and 1 as values, and call it in this case the representing function of $P$.

Under classical interpretations, which give a dichotomy of propositions into true and false, we can assign to any predicate $P$ a representing function $\pi$ which has 0 or 1 as value according as the value of $P$ is true or false; and then say that $P$ is primitive recursive if $\pi$ is.

2. General recursive functions. We shall proceed to the Herbrand-Gödel generalization of the notion of recursive function. We start with a preliminary account, certain features of which we shall then restate carefully.

The way in which the function $\phi$ is defined from the given functions in an application of one of the primitive recursive schemata amounts to this: the values $\phi\left(x_{1}, \cdots, x_{n}\right)$ of $\phi$ for the various sets $x_{1}, \cdots, x_{n}$ of arguments are determined unambiguously by the equations and the values of the given functions, using only principles of determination which we can formalize as a substitution rule and a replacement rule.

The formalization presupposes suitable conventions governing the symbolism, which are easily supplied. In particular, we must distinguish between the variables for numbers and the numerals, that is the expressions for the fixed numbers in terms of the symbols for 0 and the successor operation '. The rules are the following.

$\mathrm{R} 1$ : to substitute, for the variables $\mathrm{x}_{1}, \cdots, \mathrm{x}_{n}$ of an equation, numerals $\mathrm{x}_{1}, \cdots, \mathrm{x}_{n}$, respectively.

$\mathrm{R} 2$ : to replace a part $\mathrm{f}\left(\mathrm{x}_{1}, \cdots, \mathrm{x}_{n}\right)$ of the right member of an equation by $\mathrm{x}$, where $\mathrm{f}$ is a function symbol, where $\mathrm{x}_{1}, \cdots, \mathrm{x}_{n}, \mathrm{x}$ are numerals, and where $\mathrm{f}\left(\mathbf{x}_{1}, \cdots, \mathbf{x}_{n}\right)=\mathbf{x}$ is a given equation.

By a given equation $\mathrm{f}\left(\mathrm{x}_{1}, \cdots, \mathrm{x}_{n}\right)=\mathrm{x}$ for $\mathrm{R} 2$, we mean an equation expressing one of the values of one of the given functions for the schema application, or an equation of this form already derived by R1 and R2 from the equations of the schema application. 
Now let us consider any operation or schema, for the definition of a function in terms of given functions, which can be expressed by a system of equations determining the function values in this manner. In general the equations shall be allowed to contain, besides the principal function symbol which represents the function defined, and the given function symbols which represent the given functions, also auxiliary function symbols. The given function symbols shall not appear in the left members of the equations. Such a schema we shall call general recursive.

A function $\phi$ which can be defined from given functions $\psi_{1}, \cdots, \psi_{k}$ by a series of applications of general recursive schemata we call general recursive in the given functions; and in particular, a function $\phi$ definable ab initio by these means we call general recursive.

Suppose that a function $\phi$ is defined, either from given functions $\psi_{1}, \cdots, \psi_{k}$ or ab initio, by a succession of general recursive operations. Let us combine the successive systems of equations which effect the definition into one system, using different symbols as principal and auxiliary function symbols in each of the successive systems, and in the resulting system considering as auxiliary all of the function symbols but that representing $\phi$ and those representing $\psi_{1}, \cdots, \psi_{k}$. The restriction imposed on a general recursive schema that the given function symbols should not appear on the left will prevent any ambiguity being introduced by the interaction under $\mathrm{R} 1$ and R2 of equations in the combined system which were formerly in separate systems. Thus the definition can be considered as effected in a single general recursive operation.

In particular, any general recursive function can be defined ab initio in one operation, so that in the defining equations there are no given function symbols and what we have called the given equations for an application of R2 must all be derivable from the defining equations by previous applications of R1 and R2. For the formal development, it is convenient to adopt the convention that the principal function symbol shall be that one of the function symbols occurring in the equations of the system which comes latest in a preassigned list of function symbols. The function is then completely described by giving the system of defining equations.

We now restate the definition of general recursive function from this point of view.

A function $\phi\left(x_{1}, \cdots, x_{n}\right)$ is GENERAL RECURSIVE, if there is a system $\mathrm{E}$ of equations which defines it recursively in the following sense. A system $\mathrm{E}$ of equations defines recursively a GENERAL RECURSIVE function of $n$ variables if, for each set $x_{1}, \cdots, x_{n}$ of natural numbers, an equation of the form $\mathrm{f}\left(\mathrm{x}_{1}, \cdots, \mathrm{x}_{n}\right)=\mathrm{x}$, where $\mathrm{f}$ is the principal function symbol of $\mathrm{E}$, and where $x_{1}, \cdots, x_{n}$ are the numerals representing the natural numbers $x_{1}, \cdots, x_{n}$, is derivable from E by R1 and R2 for EXACTLY one numeral $x$. The function of $n$ variables which is defined by $\mathrm{E}$ in this case is the func- 
tion $\phi$, of which the value $\phi\left(x_{1}, \cdots, x_{n}\right)$ for $x_{1}, \cdots, x_{n}$ as arguments is THE NATURAL NUMBER $x$ REPRESENTED BY THE NUMERAL $\boldsymbol{x}$.

A predicate $P\left(x_{1}, \cdots, x_{n}\right)$ is general recursive, if there is a general recursive function $\pi\left(x_{1}, \cdots, x_{n}\right)$ taking only 0 and 1 as values such that (1) holds; in this case, $\pi$ is called the representing function of $P$. (Or, if we introduce the representing function $\pi$ first, $P$ is general recursive if $\pi$ is.)

3 . The $\mu$-operator. Consider the operator: $\mu y$ (the least $y$ such that). If this operator is applied to a predicate $R\left(x_{1}, \cdots, x_{n}, y\right)$ of the $n+1$ variables $x_{1}, \cdots, x_{n}, y$, and if this predicate satisfies the condition

$$
\left(x_{1}\right) \cdots\left(x_{n}\right)(E y) R\left(x_{1}, \cdots, x_{n}, y\right),
$$

we obtain a function $\mu y R\left(x_{1}, \cdots, x_{n}, y\right)$ of the remaining $n$ free variables $x_{1}, \cdots, x_{n}$.

Thence we have a new schema,

$$
\phi\left(x_{1}, \cdots, x_{n}\right)=\mu y\left[\rho\left(x_{1}, \cdots, x_{n}, y\right)=0\right],
$$

for the definition of a function $\phi$ from a given function $\rho$ which satisfies the condition

$$
\left(x_{1}\right) \cdots\left(x_{n}\right)(E y)\left[\rho\left(x_{1}, \cdots, x_{n}, y\right)=0\right] .
$$

We now show that this schema, subject to the condition on $\rho$, is, like (I)-(V), general recursive. For this purpose, we rewrite it in terms of equations, using an auxiliary function symbol " $\sigma$ ":

$$
\left\{\begin{aligned}
\sigma\left(0, x_{1}, \cdots, x_{n}, y\right) & =y \\
\sigma\left(z^{\prime}, x_{1}, \cdots, x_{n}, y\right) & =\sigma\left(\rho\left(x_{1}, \cdots, x_{n}, y^{\prime}\right), x_{1}, \cdots, x_{n}, y^{\prime}\right) \\
\phi\left(x_{1}, \cdots, x_{n}\right) & =\sigma\left(\rho\left(x_{1}, \cdots, x_{n}, 0\right), x_{1}, \cdots, x_{n}, 0\right) .
\end{aligned}\right.
$$

Assuming the values of $\rho$, these equations will lead us to the values of $\phi$ as defined by $\left(\mathrm{VI}_{1}\right)$, and to only those values, as follows.

Consider informally any fixed set of values of $x_{1}, \cdots, x_{n}$ (formally, this means to substitute the corresponding set of numerals for the variables " $x_{1}$ ", $\ldots$, " $x_{n}$ "). We seek to obtain the corresponding value of $\phi\left(x_{1}, \cdots, x_{n}\right)$ by replacements on the third equation, and this is the only possibility we have for obtaining that value under the two principles. First we can replace $\rho\left(x_{1}, \cdots, x_{n}, 0\right)$ by its value, and this is the only first replacement step possible on that equation. According as that value is 0 or is not 0 , we seek the value of $\sigma$ for the next replacement step from the first or second of the equations, and this is the only possible source for the next replacement value. In the first case, we obtain 0 as that value; in the second, we use the value of $\rho\left(x_{1}, \cdots, x_{n}, 1\right)$ in the second equation, and then seek another value of $\sigma$. We continue thus, with no choice in the procedure at any stage. The first case 
is first encountered when we come to use the value of $\rho\left(x_{1}, \cdots, x_{n}, y\right)$ for the first $y$ for which that value is 0 , and hence certainly for at most the $y$ given by (3). When this happens, we can complete the pending replacements to obtain that $y$ as the value of $\phi\left(x_{1}, \cdots, x_{n}\right)$. Thus we get the intended value; and because we had no choice at any stage of the procedure, we can get no other value.

The general recursiveness of the new schema is thus established. Hence, if $R\left(x_{1}, \cdots, x_{n}, y\right)$ is a general recursive predicate and (2) holds, by taking as $\rho$ the representing function of $R$, we can conclude that $\mu y R\left(x_{1}, \cdots, x_{n}, y\right)$ is a general recursive function.

What can we conclude if (2) is not assumed to hold? In this case, $\mu y R\left(x_{1}, \cdots, x_{n}, y\right)$ may not be completely defined as a function of the variables $x_{1}, \cdots, x_{n}$; but for any fixed set of values of $x_{1}, \cdots, x_{n}$, the sequence of steps by which we attempt to determine a value for $\phi\left(x_{1}, \cdots, x_{n}\right)$ from the equations remains as described for the preceding case, only with now the matter of its termination in doubt. If $(E y) R\left(x_{1}, \cdots, x_{n}, y\right)$ does hold for that set of values of $x_{1}, \cdots, x_{n}$, then it does terminate as described, with $\mu y R\left(x_{1}, \cdots, x_{n}, y\right)$ as the value; while conversely, if it does terminate, this can only be in consequence of a 0 being encountered among the values of $\rho\left(x_{1}, \cdots, x_{n}, y\right)$, so that $(E y) R\left(x_{1}, \cdots, x_{n}, y\right)$ does hold, and $\mu y R\left(x_{1}, \cdots, x_{n}, y\right)$ is the value.

Hence, in formal terms, if $\mathrm{F}$ is the system of equations obtained by adjoining, to any system $E$ which defines $\rho$ recursively, equations of the form $\left(\mathrm{VI}_{2}\right)$, with the notation so arranged that " $\phi$ " becomes the principal function symbol $f$, then: an equation of the form $f\left(x_{1}, \cdots, x_{n}\right)=x$, where $x_{1}, \cdots, x_{n}$ are the numerals representing the natural numbers $x_{1}, \cdots, x_{n}$, and where $x$ is a numeral, is derivable from $F$ by $R 1$ and $R 2$ if and only if $(E y) R\left(x_{1}, \cdots, x_{n}, y\right)$.

4. The enumeration theorem. We introduce a metamathematical predicate $\mathfrak{S}_{n}$ (for each particular $n$ ) as follows.

$\mathfrak{S}_{n}\left(\mathrm{Z}, x_{1}, \cdots, x_{n}, \mathrm{Y}\right): \mathrm{Z}$ is a system of equations, and $\mathrm{Y}$ is a formal deduction from $\mathrm{Z}$ by $\mathrm{R} 1$ and $\mathrm{R} 2$ of an equation of the form $\mathrm{f}\left(\mathrm{x}_{1}, \cdots, \mathrm{x}_{n}\right)=\mathrm{x}$, where $\mathrm{f}$ is the principal function symbol of $Z$, where $x_{1}, \cdots, x_{n}$ are the numerals representing the natural numbers $x_{1}, \cdots, x_{n}$, and where $\mathrm{x}$ is a numeral.

With this notation, we can state the last result of the preceding section symbolically:

$$
(E y) R\left(x_{1}, \cdots, x_{n}, y\right) \equiv(E \mathrm{Y}) \Im_{n}\left(\mathrm{~F}, x_{1}, \cdots, x_{n}, \mathrm{Y}\right) .
$$

From a like exploration of the possibility that the sequence of steps does not terminate, or simply from (4) by contraposition, we have also:

$$
(y) \bar{R}\left(x_{1}, \cdots, x_{n}, y\right) \equiv(\mathrm{Y}) \overline{\mathfrak{S}}_{n}\left(\mathrm{~F}, x_{1}, \cdots, x_{n}, \mathrm{Y}\right) .
$$


Using Gödel's idea of arithmetizing metamathematics $\left({ }^{6}\right)$, suppose that natural numbers have been correlated to the formal objects, distinct numbers to distinct objects. The metamathematical predicate $\mathfrak{S}_{n}\left(Z, x_{1}, \cdots, x_{n}, \mathrm{Y}\right)$ is carried by the correlation into a number-theoretic predicate $S_{n}\left(z, x_{1}, \cdots, x_{n}, y\right)$, the definition of which we complete by taking it as false for values of $z, y$ not both correlated to formal objects.

For a suitably chosen Gödel numbering, we can show, with a little trouble that $S_{n}$ is primitive recursive.

Now (4) translates under the arithmetization into

$$
(E y) R\left(x_{1}, \cdots, x_{n}, y\right) \equiv(E y) S_{n}\left(f, x_{1}, \cdots, x_{n}, y\right)
$$

with $f$ as the Gödel number of the system of equations $\mathrm{F}$. The formula

$$
(y) R\left(x_{1}, \cdots, x_{n}, y\right) \equiv(y) \bar{S}_{n}\left(g, x_{1}, \cdots, x_{n}, y\right)
$$

is obtained likewise from (5), after changing the notation so that $R$ is interchanged with $\bar{R}$.

In stating these results for reference, we shall go over from $S_{n}$ to a new predicate $T_{n}$, which entails no present disadvantage and proves to be of convenience in some further investigations $\left({ }^{7}\right)$. The predicate $T_{n}$ is defined from $S_{n}$ as follows.

$T_{n}\left(z, x_{1}, \cdots, x_{n}, y\right): S_{n}\left(z, x_{1}, \cdots, x_{n}, y\right) \&(t)\left[t<y \rightarrow \bar{S}_{n}\left(z, x_{1}, \cdots, x_{n}, t\right)\right]$.

By a theorem of Gödel $\left({ }^{8}\right)$, the primitive recursiveness of $T_{n}$ follows from that of $S_{n}$. The formulas (6) and (7) in the theorem follow from (6a) and (7a) by the definition of $T_{n}$ in terms of $S_{n}$.

THEOREM I. Given a general recursive predicate $R\left(x_{1}, \cdots, x_{n}, y\right)$, there are numbers $f$ and $g$ such that

$$
\begin{gathered}
(E y) R\left(x_{1}, \cdots, x_{n}, y\right) \equiv(E y) T_{n}\left(f, x_{1}, \cdots, x_{n}, y\right), \\
(y) R\left(x_{1}, \cdots, x_{n}, y\right) \equiv(y) \bar{T}_{n}\left(g, x_{1}, \cdots, x_{n}, y\right) .
\end{gathered}
$$

Now $(E y) T_{n}\left(z, x_{1}, \cdots, x_{n}, y\right)$ is a fixed predicate of the form $(E y) R\left(z, x_{1}, \cdots, x_{n}, y\right)$ where $R$ is general recursive (in fact, as it happens, primitive recursive). By the theorem, if we take successively $z=0,1,2, \cdots$, we obtain an enumeration (with repetitions) of all predicates of the form $(E y) R\left(x_{1}, \cdots, x_{n}, y\right)$ where $R$ is general recursive $\left({ }^{9}\right)$. Likewise, the theorem gives us a fixed predicate of the form $(y) R\left(z, x_{1}, \cdots, x_{n}, y\right)$ where $R$ is general recursive which enumerates all predicates of the form $(y) R\left(x_{1}, \cdots, x_{n}, y\right)$

(6) Gödel [1].

(7) A revision, April 13, 1942.

(8) Gödel [1, IV].

(9) This result entered partly into the last theorem of Kleene [2], but the advantage of using it at an earlier stage was overlooked. In anticipation, we may remark that XI-XVI of that paper are essentially special cases of Theorem II below (with now a constructive proof for XVI). 
where $R$ is general recursive. These enumerations form the basis for the application of Cantor's diagonal method in the next section.

5. The general theorem. By a familiar rule of classical logic, in each of the following pairs of propositions (with a fixed $R$ for a given pair),

$$
\begin{aligned}
(E x) R(x) & (x)(E y) R(x, y) & (E x)(y)(E z) R(x, y, z) & \cdots \\
(x) \bar{R}(x) & (E x)(y) \bar{R}(x, y) & (x)(E y)(z) \bar{R}(x, y, z) & \cdots,
\end{aligned}
$$

either member is equivalent to the negation of the other. Hence we may assert non-equivalence between the members of the pair. This argument is not good in the intuitionistic logic. However, the non-equivalence for the case of one quantifier,

$$
(E x) R(x) \not \equiv(x) \bar{R}(x)
$$

does hold good intuitionistically.

Consider the predicate form $(x) R(a, x)$ where $R$ is general recursive. This gives a particular predicate of the variable $a$, whenever we specify the general recursive predicate $R(a, x)$ of two variables. In particular, $(x) \bar{T}_{1}(a, a, x)$ is a predicate of this form.

We shall show that this predicate is neither general recursive nor expressible in the form $(E x) R(a, x)$ where $R$ is general recursive.

For this purpose, suppose we have selected any particular general recursive $R(a, x)$, giving a particular predicate of the latter form. By (6), there is for this $R$ a number $f$ such that

$$
(E x) R(a, x) \equiv(E x) T_{1}(f, a, x)
$$

Substituting the number $f$ for the variable $a$,

$$
(E x) R(f, x) \equiv(E x) T_{1}(f, f, x)
$$

By (8),

$$
(E x) T_{1}(f, f, x) \not \equiv(x) \bar{T}_{1}(f, f, x) .
$$

Combining (10) and (11),

$$
(E x) R(f, x) \not \equiv(x) \bar{T}_{1}(f, f, x) .
$$

This refutes, for $a=f$, the equivalence of $(E x) R(a, x)$ to $(x) \bar{T}_{1}(a, a, x)$. Since this refutation can be effected, whatever general recursive $R$ we chose, for some $f$ depending on the $R$, the predicate $(x) \bar{T}_{1}(a, a, x)$ is not expressible in the form $(E x) R(a, x)$ where $R$ is general recursive.

A fortiori, $(x) \bar{T}_{1}(a, a, x)$ is not expressible in the form $R(a)$ where $R$ is general recursive. For were it so expressed, we should then have it in the form $(E x) R(a, x)$ where $R$ is general recursive, by taking as $R(a, x)$ the predicate $R(a) \& x=x$.

This completes the proof of one case of the next theorem. 
For another case, consider the predicate form $(E x) R(a, x)$ where $R$ is general recursive. We can show similarly, using (7) instead of (6), that the predicate $(E x) T_{1}(a, a, x)$, which has this form, is neither general recursive nor expressible in the form $(x) R(a, x)$ where $R$ is general recursive.

To illustrate the treatment of a case with more than one quantifier, consider the predicate form $(x)(E y)(z) R(a, x, y, z)$ where $R$ is general recursive. The predicate $(x)(E y)(z) \bar{T}_{3}(a, a, x, y, z)$ has this form. Select any particular general recursive $R(a, x, y, z)$. By (6), for some $f$ depending on this $R$,

$$
(E z) R(a, x, y, z) \equiv(E z) T_{3}(f, a, x, y, z) .
$$

By corresponding quantifications of these equivalent predicates,

$$
(E x)(y)(E z) R(a, x, y, z) \equiv(E x)(y)(E z) T_{3}(f, a, x, y, z) .
$$

Classically, we can complete the argument as before, showing that $(x)(E y)(z) \bar{T}_{3}(a, a, x, y, z)$ is not expressible in any of the forms

$$
\begin{array}{rlll}
R(a) & (E x) R(a, x) & (x)(E y) R(a, x, y) & (E x)(y)(E z) R(a, x, y, z) \\
& (x) R(a, x) & (E x)(y) R(a, x, y)
\end{array}
$$

where the $R$ for the form is general recursive.

To obtain an alternative phrasing of the theorem, in which it holds for all cases intuitionistically, we may omit in the classical proof the step which interchanges the two kinds of quantifiers under the operation of negation. We thus show that the predicates $(\overline{E x}) T_{1}(a, a, x), \overline{(x)} \bar{T}_{1}(a, a, x)$, $(\overline{E x})(y)(E z) T_{3}(a, a, x, y, z)$, and so on, are neither expressible in the respective forms $(E x) R(a, x),(x) R(a, x),(E x)(y)(E z) R(a, x, y, z)$, and so on, where $R$ is general recursive, nor in any of the forms with fewer quantifiers.

THEOREM II. Classically, and for the one-quantifier forms intuitionistically: To each of the forms

$$
R(a) \begin{array}{rlr}
(E x) R(a, x) & (x)(E y) R(a, x, y) & (E x)(y)(E z) R(a, x, y, z) \cdots \\
(x) R(a, x) & (E x)(y) R(a, x, y) & (x)(E y)(z) R(a, x, y, z) \cdots
\end{array}
$$

where the $R$ for each is general recursive, after the first, there is a predicate expressible in that form but not in the other form with the same number of quantifiers nor in any of the forms with fewer quantifiers.

Classically, and intuitionistically: To each of the forms, after the first, there is a predicate expressible in the negation of that form but not in that form itself nor in any of the forms with fewer quantifiers.

For simplicity, we have given the theorem for predicates of one variable $a$, but it holds:

Likewise, replacing the variable a throughout by $n$ variables $a_{1}, \cdots, a_{n}$, for any fixed positive integer $n$. 
By an elementary predicate, we shall mean one which is expressible in terms of general recursive predicates, the operations $\&, \vee,-, \rightarrow$, $\equiv$ of the propositional calculus, and quantifiers.

Suppose given an expression for a predicate in these terms. By the classical predicate calculus, we can transform the expression so that all quantifiers stand at the front. For each $m$, let $(x)_{1}, \cdots,(x)_{m}$ be a set of $m$ primitive recursive functions of $x$ which as a set ranges, with or without repetitions, over all $m$-tuples of natural numbers, as $x$ ranges over all natural numbers (such sets of functions are known). The equivalences

$$
\begin{aligned}
\left(E x_{1}\right) \cdots\left(E x_{m}\right) A\left(x_{1}, \cdots, x_{m}\right) & \equiv(E x) A\left((x)_{1}, \cdots,(x)_{m}\right), \\
\left(x_{1}\right) \cdots\left(x_{m}\right) A\left(x_{1}, \cdots, x_{m}\right) & \equiv(x) A\left((x)_{1}, \cdots,(x)_{m}\right)
\end{aligned}
$$

enable us to eliminate consecutive occurrences of like quantifiers. These transformations leave as operand of the prefixed quantifiers a general recursive predicate of the free and bound variables. Hence, classically, the predicate forms listed in the theorem for a given $n$ suffice for the expression of every elementary predicate of $n$ variables.

The theorem then says that no finite sublist of the forms would suffice.

Classically, we are led to a classification of the elementary predicates according to the minimum numbers of quantifiers which would suffice for their expression in terms of general recursive predicates and quantifiers.

The analogy between the logical operations of existential and universal quantification and geometrical operations of projection and intersection, respectively, is well known $\left({ }^{10}\right)$. The possibility of a connection between present results and theories of Borel and Baire is suggested( $\left.{ }^{11}\right)$.

\section{Primitive, general, and partial Recursive PREDICATES UNDER QUANTIFICATION}

6. Partial recursive functions. The author's definition of partial recursive function extends the Herbrand-Gödel definition of general recursive function to functions $\phi$ of $n$ variables which need not be defined for all $n$-tuples of natural numbers as arguments, retaining the characteristic of that definition with respect to each $n$-tuple for which the function is defined( $\left.{ }^{12}\right)$. The partial recursive functions include the general recursive functions as those which are defined for all sets of arguments.

For a more complete description, take the definition of general recursive function which is given at the end of $\$ 2$, and replace the four capitalized phrases by the following, respectively: PARTIAL RECURSIVE; PARTIAL RECURSIVE; AT MOST; THE NATURAL NUMBER $x$ REPRE-

(10) In particular, it has been discussed by Tarski.

(ii) This suggestion was made to the author by Gödel and by Ulam.

(12) Kleene [4]. 


\section{SENTED BY THE NUMERAL $x$ IF THAT NUMERAL EXISTS, AND IS OTHERWISE UNDEFINED.}

In dealing with functions which may not be completely defined, we interpret the equation $\phi\left(x_{1}, \cdots, x_{n}\right)=\psi\left(x_{1}, \cdots, x_{n}\right)$ as the assertion that $\phi$ and $\psi$ have the same value for $x_{1}, \cdots, x_{n}$ as arguments, taking it as undefined (nonsignificant) if either value is undefined. We write $\phi\left(x_{1}, \cdots, x_{n}\right) \simeq \psi\left(x_{1}, \cdots, x_{n}\right)$ to express the assertion that, if either of $\phi$ and $\psi$ is defined for the arguments $x_{1}, \cdots, x_{n}$, the other is and the values are the same, and if either of $\phi$ and $\psi$ is undefined for those arguments, the other is.

Similarly, in dealing with predicates which may not be completely defined, $P\left(x_{1}, \cdots, x_{n}\right) \equiv Q\left(x_{1}, \cdots, x_{n}\right)$ expresses equivalence of value, and is undefined if the value of either member is undefined; while $P\left(x_{1}, \cdots, x_{n}\right)$ $\cong Q\left(x_{1}, \cdots, x_{n}\right)$ expresses that the definition of either implies mutual definition with equivalence, and the indefinition of either implies mutual indefinition.

A predicate $P\left(x_{1}, \cdots, x_{n}\right)$ not necessarily defined for all $n$-tuples of natural numbers as arguments is partial recursive, if there is a partial recursive function $\pi\left(x_{1}, \cdots, x_{n}\right)$ taking only 0 and 1 as values such that

$$
P\left(x_{1}, \cdots, x_{n}\right) \cong \pi\left(x_{1}, \cdots, x_{n}\right)=0 ;
$$

in this case, $\pi$ is called the representing function of $P$. (Or if we first introduce a representing function $\pi$ of $P$, the value of which is to be 0,1 , or undefined according as the value of $P$ is true, false, or undefined, then $P$ is partial recursive if $\pi$ is.)

In $\S \S 2,3$, we remarked the general recursiveness of Schemata (I)-(VI) with (VI) subjected to the condition (3); and we also considered Schema (VI) for the case that $\rho$ is general recursive but (3) is not required to hold. The method of those sections applies equally well without the restrictions; in explanation of the schemata when the given functions may not be completely defined or (3) not hold for (VI), it will suffice here to remark that the conditions of definition for the functions introduced by the schemata may be inferred a posteriori from the metamathematical results.

THEOREM III. The class of general recursive functions is closed under applications of Schemata (I)-(VI) with (3) holding for applications of (VI).

The class of partial recursive functions is closed under applications of Schemata (I)-(VI).

COROLlaRY. Every function obtainable by applications of Schemata (I)-(VI) with (3) holding for applications of (VI) is general recursive.

Every function obtainable by applications of Schemata (I)-(VI) is partial recursive.

7. Normal form for recursive functions. We shall pursue a little further 
the method of $\$ 4$ to obtain the converse of this result. Besides the metamathematical predicate $\mathfrak{S}_{n}$, we now require a metamathematical function as follows.

$\mathfrak{u}(\mathrm{Y})$ : the natural number $x$ which the numeral $\mathrm{x}$ represents, in case $\mathrm{Y}$ is a formal deduction of an equation of the form $\mathrm{t}=\mathrm{x}$, where $\mathrm{x}$ is a numeral and $\mathrm{t}$ is any term; and 0, otherwise.

According to the definition of general recursive function, if $\phi$ is a general recursive function of $n$ variables, there is a system $\mathrm{E}$ of equations such that

$$
\begin{gathered}
\left(x_{1}\right) \cdots\left(x_{n}\right)(E \mathrm{Y}) \Im_{n}\left(\mathrm{E}, x_{1}, \cdots, x_{n}, \mathrm{Y}\right) \\
\left(x_{1}\right) \cdots\left(x_{n}\right)(\mathrm{Y})\left[\Im_{n}\left(\mathrm{E}, x_{1}, \cdots, x_{n}, \mathrm{Y}\right) \rightarrow \mathfrak{U}(\mathrm{Y})=\phi\left(x_{1}, \cdots, x_{n}\right)\right]
\end{gathered}
$$

and the function $\phi\left(x_{1}, \cdots, x_{n}\right)$ can be expressed in terms of $E$ thus

$$
\phi\left(x_{1}, \cdots, x_{n}\right)=\mathfrak{U}\left(\mu \mathrm{Y} \Im_{n}\left(\mathrm{E}, x_{1}, \cdots, x_{n}, \mathrm{Y}\right)\right),
$$

if we understand the formal objects to be enumerated in some order, so that the operator $\mu$ can be applied with respect to the metamathematical variable $\mathrm{Y}$; we may take the order to be that of the corresponding Gödel numbers.

If $\phi$ is a partial recursive function of $n$ variables, instead of asserting (18), we can write

$$
(E \mathrm{Y}) \Im_{n}\left(\mathrm{E}, x_{1}, \cdots, x_{n}, \mathrm{Y}\right)
$$

as the condition on $x_{1}, \cdots, x_{n}$ that the function be defined for $x_{1}, \cdots, x_{n}$ as arguments; we have (19), taking the implication to be true whenever the first member is false, irrespective of the status of the second member; and our convention calls for rewriting (20) thus,

$$
\phi\left(x_{1}, \cdots, x_{n}\right) \simeq \mathfrak{u}\left(\mu \mathrm{Y} \Im_{n}\left(\mathrm{E}, x_{1}, \cdots, x_{n}, \mathrm{Y}\right)\right),
$$

in order that it be true (and not sometimes undefined) for all values of $x_{1}, \cdots, x_{n}$.

By the Gödel numbering already considered, the metamathematical function $\mathfrak{U}(\mathrm{Y})$ is carried into a number-theoretic function $U(y)$, the definition of which we complete by taking the value to be 0 for any $y$ not correlated to a formal object. If the Gödel numbering was suitably chosen, $U$ as well as $S_{n}$ is primitive recursive.

Now (20), (18) and (19) in terms of $\mathfrak{S}_{n}$ and $\mathfrak{U}$ are carried into formulas of like form in terms of $S_{n}$ and $U$. On passing over from $S_{n}$ to $T_{n}$, we then have the (22), (23) and (24) of the theorem $\left({ }^{13}\right)$. The part of the theorem which refers to a partial recursive function is obtained similarly.

THEOREM IV. Given a general recursive function $\phi\left(x_{1}, \cdots, x_{n}\right)$, there is a

(13) Kleene [2, IV], with some changes in the formulation. The present $S_{n}$ corresponds to the former $T_{n}$, using the Gödel numbering of proofs instead of the enumeration of provable equations. 
number e such that

$$
\begin{gathered}
\phi\left(x_{1}, \cdots, x_{n}\right)=U\left(\mu y T_{n}\left(e, x_{1}, \cdots, x_{n}, y\right)\right), \\
\left(x_{1}\right) \cdots\left(x_{n}\right)(E y) T_{n}\left(e, x_{1}, \cdots, x_{n}, y\right), \\
\left(x_{1}\right) \cdots\left(x_{n}\right)(y)\left[T_{n}\left(e, x_{1}, \cdots, x_{n}, y\right) \rightarrow U(y)=\phi\left(x_{1}, \cdots, x_{n}\right)\right] .
\end{gathered}
$$
that

$$
\phi\left(x_{1}, \cdots, x_{n}\right) \simeq U\left(\mu y T_{n}\left(e, x_{1}, \cdots, x_{n}, y\right)\right)
$$

where

$$
(E y) T_{n}\left(e, x_{1}, \cdots, x_{n}, y\right)
$$

is the condition of definition of the function, and (24) holds.

Thus any general recursive function (any partial recursive function) is expressible in the form $\psi\left(\mu y R\left(x_{1}, \cdots, x_{n}, y\right)\right)$ with (2) holding (in the form $\left.\psi\left(\mu y R\left(x_{1}, \cdots, x_{n}, y\right)\right)\right)$ where $\psi$ and $R$ are primitive recursive. Hence:

COROLlARY. Every general recursive function is obtainable by applications of Schemata (I)-(VI) with (3) holding for applications of (VI).

Every partial recursive function is obtainable by applications of Schemata (I)-(VI).

Formula (25) contains the substance of the theorem. For it implies the condition of definition of the function; and, in the case that $\phi\left(x_{1}, \cdots, x_{n}\right)$ is defined for all sets of arguments, it gives (22) and (23). Moreover by the definition of $T_{n}$ in terms of $S_{n}$, it implies (24).

We say that $e$ defines $\phi$ recursively, or $e$ is a Gödel number of $\phi$, if (25) holds $\left({ }^{14}\right)$, in which case $e$ has all the properties in relation to $\phi$ which are specified in the theorem.

It is here that the advantage of using $T_{n}$ instead of $S_{n}$ appears. A number $e$ which satisfies $\phi\left(x_{1}, \cdots, x_{n}\right) \simeq U\left(\mu y S_{n}\left(e, x_{1}, \cdots, x_{n}, y\right)\right.$ ) (which is equivalent to $(25))$ does not necessarily satisfy $\left(x_{1}\right) \cdots\left(x_{n}\right)(y)\left[S_{n}\left(e, x_{1}, \cdots, x_{n}, y\right)\right.$ $\left.\rightarrow U(y)=\phi\left(x_{1}, \cdots, x_{n}\right)\right]$. While we could get around the difficulty by imposing the latter as an additional condition on the Gödel numbers, it is more convenient simply to use $T_{n}$ instead of $S_{n}$. (On the basis of Theorem III and the results which we had in terms of $S_{n}$ before passing over to $T_{n}$, one can set up a primitive recursive function $V$ such that, if $e$ satisfies (25), then $V(e)$ has all the properties in terms of $S_{n}$.)

The numbers $f$ and $g$ for Theorem I can be described now as any numbers which define recursively the partial recursive functions $\mu y R\left(x_{1}, \cdots, x_{n}, y\right)$ and $\mu y \bar{R}\left(x_{1}, \cdots, x_{n}, y\right)$, respectively.

(14) Kleene [2, Definition 2c, p. 738] and [4, top p. 153]. We have now also the changes in the formulation of Theorem IV. 
8. Consistency. Let us review the arguments used in proof of Theorems I and III. For rigor, these have to be put in metamathematical form. Let $E$ be the system of equations associated with a series of applications of Schemata (I)-(VI). We shall review only the case that no given function symbols occur in $\mathrm{E}$.

In general, we easily establish that, for each of certain sets $x_{1}, \cdots, x_{n}$ of natural numbers, an equation of the form $\mathrm{f}\left(\mathrm{x}_{1}, \cdots, \mathrm{x}_{n}\right)=\mathrm{x}$, as described in the definitions of general and partial recursive function, is derivable from $\mathrm{E}$ by $\mathrm{R} 1$ and $\mathrm{R} 2$. In particular, if we are proving that $\mathrm{E}$ defines a general recursive function, we must show this for all $x_{1}, \cdots, x_{n}$; if we have a prior interpretation of the schemata applications as definition of a (partial or complete) function $\phi\left(x_{1}, \cdots, x_{n}\right)$, or require that $\mathrm{E}$ define a $\phi\left(x_{1}, \cdots, x_{n}\right)$ already known to us in some other manner, we must show this for all $x_{1}, \cdots, x_{n}$ belonging to the range of definition of $\phi$, and also show that the $\mathrm{x}$ in the equation is the numeral representing the value of $\phi$ for $x_{1}, \cdots, x_{n}$ as arguments. This property of the equations $\mathrm{E}$ and rules $\mathrm{R} 1$ and $\mathrm{R} 2$, the precise formulation of which depends on the circumstances, we call the "completeness property." (When we wish merely to show that E defines a partial recursive function, the function to be determined a posteriori from $\mathrm{E}$, no completeness property is required.)

The second part of the discussion consists in showing that an equation of the described form $f\left(x_{1}, \cdots, x_{n}\right)=x$ is derivable from $E$ for at most one numeral $\mathbf{x}$; or if we have already established completeness in one of the above senses, that the equations $f\left(x_{1}, \cdots, x_{n}\right)=x$ referred to in the discussion of completeness, for various $x_{1}, \cdots, x_{n}$, are the only equations of that form which are derivable from E by $\mathrm{R} 1$ and R2. This we call the "consistency property."

As we indicated in $\$ 2$, it suffices to handle each of the schemata in turn, assuming equations for use with $\mathrm{R} 2$ which give the values of the given functions. The argument for consistency which we sketched in $\$ 3$ for Schema (VI) applies as well to the other schemata. For Schema (IV) there is indeed a choice in the order in which the values of the several $\chi$ 's are introduced, but it is without effect on the final result.

This very easy consistency proof was gained by restricting the replacement rule so that replacement is only performable on the right member of an equation, a part $\mathrm{f}\left(\mathrm{x}_{1}, \cdots, \mathrm{x}_{n}\right)$ where $\mathrm{f}$ is a function symbol and $x_{1}, \cdots, \mathrm{x}_{n}$ are numerals being replaced by a numeral $x$. This eliminates the possibility of deriving an equation of the form $\mathrm{g}\left(y_{1}, \cdots, y_{m}\right)=y$, where $g$ is a fixed function symbol, $y_{1}, \cdots, y_{m}$ are fixed numerals, and $y$ is any numeral, along essentially different paths within the system, and therewith the possibility that such an equation should be derivable for different $y$ 's.

In some previous versions of the theories of general and partial recursive functions, the replacement rule was not thus restricted. The consistency proof 
which we gave in the version with the unrestricted replacement rule was based on the notion of verifiability of an equation $\left({ }^{15}\right)$. This notion makes presupposition of the values of the functions, and for the theory of partial recursive functions also of the determinateness whether or not the values are defined. In the latter case, it is not finitary. To give a constructive consistency proof for the theory of partial recursive functions with the stronger replacement rule seems to require the type of argument used in the Church-Rosser consistency proof for $\lambda$-conversion $\left({ }^{16}\right)$, and in the Ackermann-von Neumann consistency proof for a certain part of number theory in terms of the Hilbert $\epsilon$-symbol $\left({ }^{17}\right)$.

It is easily shown, by using the method of proof of Theorem IV to obtain the same normal form with the stronger replacement rule, that every function partial recursive under the stronger replacement rule is such under the weaker.

Thus we find the curious fact that the main difficulty in showing the equivalence of the two notions of recursiveness comes in showing that the stronger rule suffices to define as many functions as the weaker. This is because the consistency of a stronger formalism is involved. The consistency of that formalism is of interest on its own account, but is extraneous for the theory of recursive definition, including the applications corresponding to those of Church in terms of the $\lambda$-notation which presuppose the complicated ChurchRosser consistency proof. All that is required for the theory of recursive definition is some consistent formalism sufficient for the derivation of the equations giving the values of the functions.

To this discussion we may add several supplementary remarks. We might in practice have a system $E$ of equations and a method for deriving from $E$ by $\mathrm{R} 1$ and the strong replacement rule, for all and only the $n$-tuples of a certain set, an equation of the form $\mathrm{f}\left(\mathrm{x}_{1}, \cdots, \mathbf{x}_{n}\right)=\mathbf{x}$ with a determinate $\mathbf{x}$, but lack the knowledge that unlimited use of the two rules could not lead to other such equations. In this situation, a function is defined intuitively for the $n$-tuples of the set, and undefined off the set. If we can characterize metamathematically our method of applying the two rules, we shall obtain a limited formalism known to be consistent, and the method used in establishing Theorem IV can then be applied to obtain equations defining the function recursively with the weak replacement rule.

For some types of equations which define a function recursively with the strong replacement rule (consistency being known), a more direct method may be available for obtaining a system defining the function recursively with the weak replacement rule. For example, consider (in informal language) the equation $\phi(\psi(x))=\chi(x)$. To use this in deriving equations giving values of $\phi$, we need to introduce values of $\psi$ by replacement on the left. After

(15) Kleene $[2$, p. 731$]$ and $[4, \S 2$, the bracketed portion of the fifth paragraph].

(16) Church and Rosser [1].

(17) Hilbert and Bernays [1, §2, part 4, pp. 93-130, and Supplement II, pp. 396-400]. 
expressing the equation in the form $\phi(y)=\left(\mu w\left[\psi\left((w)_{1}\right)=y \& \chi\left((w)_{1}\right)=(w)_{2}\right]\right)_{2}$, and separating the latter into a series of equations without the $\mu$-symbol by the method which the theory of the schemata affords, replacement will be required only on the right. This device is applicable to any equation of which the left member has the form $\mathrm{f}\left(\mathrm{g}_{1}\left(\mathrm{x}_{1}, \cdots, \mathrm{x}_{n}\right), \cdots, \mathrm{g}_{m}\left(\mathrm{x}_{1}, \cdots, \mathrm{x}_{n}\right)\right)$.

The precise form of the restriction which is used to weaken the replacement rule is somewhat arbitrary, so long as it accomplishes its purpose of channelling the deductions of equations giving the values of the functions. The restriction as it was stated in the early Gödel version is now simplified, since we need to consider only equations having the forms appearing in the six schemata. Gödel provided for equations the left members of which could have the form $f\left(g_{1}\left(x_{1}, \cdots, x_{n}\right), \cdots, g_{m}\left(x_{1}, \cdots, x_{n}\right)\right)$ where $f$ is the principal function symbol and $g_{1}, \cdots, g_{m}$ are given function symbols, and therefore allowed replacement on the left in the case of the g's.

9. Predicates expressible in both one-quantifier forms. By Theorem IV, for any general recursive predicate $P\left(x_{1}, \cdots, x_{n}\right)$,

$$
\begin{aligned}
& P\left(x_{1}, \cdots, x_{n}\right) \equiv(E y)\left[T_{n}\left(e, x_{1}, \cdots, x_{n}, y\right) \& U(y)=0\right], \\
& P\left(x_{1}, \cdots, x_{n}\right) \equiv(y)\left[T_{n}\left(e, x_{1}, \cdots, x_{n}, y\right) \rightarrow U(y)=0\right],
\end{aligned}
$$

where $e$ is any Gödel number of the representing function of $P$.

Conversely, suppose that for a predicate $P$ both $P\left(x_{1}, \cdots, x_{n}\right)$ $\equiv(E y) R\left(x_{1}, \cdots, x_{n}, y\right)$ and $P\left(x_{1}, \cdots, x_{n}\right) \equiv(y) S\left(x_{1}, \cdots, x_{n}, y\right)$ where $R$ and $S$ are general recursive. From the second of these equivalences, under classical interpretations, $\bar{P}\left(x_{1}, \cdots, x_{n}\right) \equiv(E y) \bar{S}\left(x_{1}, \cdots, x_{n}, y\right)$. By the classical law of the excluded middle, $(E y)\left[R\left(x_{1}, \cdots, x_{n}, y\right) \bigvee \bar{S}\left(x_{1}, \cdots, x_{n}, y\right)\right]$. Therefore

$$
P\left(x_{1}, \cdots, x_{n}\right) \equiv R\left(x_{1}, \cdots, x_{n}, \mu y\left[R\left(x_{1}, \cdots, x_{n}, y\right) \vee \bar{S}\left(x_{1}, \cdots, x_{n}, y\right)\right]\right),
$$

where the second member is general recursive by Theorem III.

TheOREM V. Every general recursive predicate $P\left(x_{1}, \cdots, x_{n}\right)$ is expressible in both of the forms $(E y) R\left(x_{1}, \cdots, x_{n}, y\right)$ and $(y) R\left(x_{1}, \cdots, x_{n}, y\right)$ where the $R$ for each is primitive recursive. Under classical interpretations, conversely, every predicate expressible in both of these forms where the $R$ for each is general recursive is general recursive.

Now consider any predicate expressible in one of the forms of Theorem II after the first. According as the innermost quantifier in this form is existential or universal, we can apply (26) or (27), and then absorb the extra quantifier by (15) or (16), respectively, to obtain the original form but with a primitive recursive $R$. For example,

$$
\begin{aligned}
(x)(E y) R(a, x, y) & \equiv(x)\left(E y_{1}\right)\left(E y_{2}\right)\left[T_{3}\left(e, a, x, y_{1}, y_{2}\right) \& U\left(y_{2}\right)=0\right] \\
& \equiv(x)(E y)\left[T_{3}\left(e, a, x,(y)_{1},(y)_{2}\right) \& U\left((y)_{2}\right)=0\right] .
\end{aligned}
$$


CoROllary. The class of predicates expressible in a given one of the forms of Theorem II after the first (for a given $n$ variables) is the same whether a primitive recursive or a general recursive $R$ be allowed.

This generalizes the observation of Rosser that a class enumerable by a general recursive function is also enumerable by a primitive recursive func$\operatorname{tion}\left({ }^{18}\right)$.

The formulas for the one-quantifier cases are

$$
\begin{aligned}
& (E y) R\left(x_{1}, \cdots, x_{n}, y\right) \\
& \quad \equiv(E y)\left[T_{n+1}\left(e, x_{1}, \cdots, x_{n},(y)_{1},(y)_{2}\right) \& U\left((y)_{2}\right)=0\right], \\
& \begin{array}{r}
(y) R\left(x_{1}, \cdots,\right. \\
\left.\quad x_{n}, y\right) \\
\quad \equiv(y)\left[T_{n+1}\left(e, x_{1}, \cdots, x_{n},(y)_{1},(y)_{2}\right) \rightarrow U\left((y)_{2}\right)=0\right],
\end{array}
\end{aligned}
$$

where $e$ is any Gödel number of the representing function of $R$. These afford a new proof of the enumeration theorem of $\$ 4$, with new enumerating predicates, and thence a new proof of Theorem II.

10. Partial recursive predicates. Let $P\left(x_{1}, \cdots, x_{n}\right)$ be a predicate which may not be defined for all $n$-tuples of natural numbers as arguments. By a completion of $P$ we understand a predicate $Q$ such that, if $P\left(x_{1}, \cdots, x_{n}\right)$ is defined, then $Q\left(x_{1}, \cdots, x_{n}\right)$ is defined and has the same value, and if $P\left(x_{1}, \cdots, x_{n}\right)$ is undefined, then $Q\left(x_{1}, \cdots, x_{n}\right)$ is defined. In particular, the completion $P^{+}\left(x_{1}, \cdots, x_{n}\right)$ which is false when $P\left(x_{1}, \cdots, x_{n}\right)$ is undefined, and the completion $P-\left(x_{1} ; \cdots, x_{n}\right)$ which is true when $P\left(x_{1}, \cdots, x_{n}\right)$ is undefined, we call the positive completion and negative completion of $P\left(x_{1}, \cdots, x_{n}\right)$, respectively. (In $P$ and $P^{+}$, the "positive parts" coincide; in $P$ and $P^{-}$, the "negative parts" coincide.)

If $P\left(x_{1}, \cdots, x_{n}\right)$ is a partial recursive predicate, then by Theorem IV,

$$
\begin{aligned}
& P^{+}\left(x_{1}, \cdots, x_{n}\right) \equiv(E y)\left[T_{n}\left(e, x_{1}, \cdots, x_{n}, y\right) \& U(y)=0\right], \\
& P^{-}\left(x_{1}, \cdots, x_{n}\right) \equiv(y)\left[T_{n}\left(e, x_{1}, \cdots, x_{n}, y\right) \rightarrow U(y)=0\right],
\end{aligned}
$$

where $e$ is any Gödel number of the representing function of $P$.

Conversely, if $R\left(x_{1}, \cdots, x_{n}, y\right)$ is any general recursive predicate, then by Theorem III,

$$
\begin{aligned}
(E y) R\left(x_{1}, \cdots, x_{n}, y\right) & \equiv \mu y R\left(x_{1}, \cdots, x_{n}, y\right)={ }^{+} \mu y R\left(x_{1}, \cdots, x_{n}, y\right), \\
(y) R\left(x_{1}, \cdots, x_{n}, y\right) & \equiv \mu y \bar{R}\left(x_{1}, \cdots, x_{n}, y\right) \neq{ }^{-} \mu y \bar{R}\left(x_{1}, \cdots, x_{n}, y\right) .
\end{aligned}
$$

TheOREM VI. The positive completion $P^{+}\left(x_{1}, \cdots, x_{n}\right)$ of a partial recursive predicate $P\left(x_{1}, \cdots, x_{n}\right)$ is expressible in the form $(E y) R\left(x_{1}, \cdots, x_{n}, y\right)$ where $R$ is primitive recursive; and conversely, any predicate expressible in the form $(E y) R\left(x_{1}, \cdots, x_{n}, y\right)$ where $R$ is general recursive is the positive completion $P^{+}\left(x_{1}, \cdots, x_{n}\right)$ of a partial recursive predicate $P\left(x_{1}, \cdots, x_{n}\right)$.

(18) Rosser [1, Lemma I, Corollary I, p. 88]. 
Dually, for negative completions $P^{-}\left(x_{1}, \cdots, x_{n}\right)$ and the predicate form $(y) R\left(x_{1}, \cdots, x_{n}, y\right)$.

It follows that, for the predicate forms of Theorem II which have an existential quantifier (universal quantifier) innermost, we may, without altering the class of predicates expressible in that form, take $R$ to be the positive completion (negative completion) of a partial recursive predicate.

Let us abbreviate $U\left(\mu y T_{n}\left(z, x_{1}, \cdots, x_{n}, y\right)\right)$ as $\Phi_{n}\left(z, x_{1}, \cdots, x_{n}\right)\left({ }^{19}\right)$. Then $\Phi_{n}$ is a fixed partial recursive function of $n+1$ variables, from which any partial recursive function $\phi$ of $n$ variables can be obtained thus (rewriting (25)),

$$
\phi\left(x_{1}, \cdots, x_{n}\right) \simeq \Phi_{n}\left(e, x_{1}, \cdots, x_{n}\right)
$$

where $e$ is any Gödel number of $\phi$. Since for a constant $z, \Phi_{n}\left(z, x_{1}, \cdots, x_{n}\right)$ is always a partial recursive function of the remaining $n$ variables, $\Phi_{n}\left(z, x_{1}, \cdots, x_{n}\right)$ therefore gives for $z=0,1,2, \cdots$ an enumeration (with . repetitions) of the partial recursive functions of $n$ variables. It follows that $\Phi_{n}\left(z, x_{1}, \cdots, x_{n}\right)=0$ is a partial recursive predicate of $n+1$ variables which enumerates (with repetitions) the partial recursive predicates of $n$ variables.

This, seen in the light of Theorem VI, has as consequence the enumeration theorem of $\$ 2$ (with other enumerating predicates), and thence by Cantor's diagonal method Theorem II.

Elsewhere, the enumeration theorem for partial recursive functions gave by Cantor's diagonal method what may be called the fundamental theorem for proofs of recursive definability $\left({ }^{20}\right)$.

This fundamental theorem, and the existence of partial recursive functions and predicates, no completions of which are general recursive $\left({ }^{21}\right)$, are what occasioned the introduction of the notion of a partial recursive function.

\section{INCOMPLETENESS THEOREMS IN THE FOUNDATIONS OF NUMBER THEORY}

11. Introductory remarks. We entertain various propositions about natural numbers. These propositions have meaning, independently of or prior to the consideration of formal postulates and rules of proof. We pose the problem of systematizing our knowledge about these propositions into a theory of some kind. For certain definitions of our objectives in constructing the theory, and certain classes of propositions, we shall be able to reach definite answers concerning the possibility of constructing the theory.

The naive informal approach which we are adopting may be contrasted

(19) Using the notation of Kleene [4, bottom p. 152], but with the changes in the formulation of Theorem IV.

(20) Kleene $[4$, the last result in $\$ 2]$.

(21) Kleene [4, Footnote 3]. 
with that form of the postulational approach which consists in first listing formal postulates, which are then said to define the content of the theory based on them. In the case of number theory, the formal approach cannot render entirely dispensable an intuitive understanding of propositions of the kind which we commonly interpret the theory to be about. For the explicit statement of the postulates and characterization of the manner in which they are to determine the theory belong to a metatheory on another level of discourse; and the ultimate metatheory must be an intuitive mathematics unregulated by explicit postulates, and having the essential character of number theory.

Of course the informality of our investigation does not preclude the enumeration, from another level, of postulates which would suffice to describe it. Indeed, such regulation may perhaps be considered necessary from an intuitive standpoint for that part of it which belongs to the context of classical mathematics.

The propositions about natural numbers which we shall consider will contain parameters. We shall thus have infinitely many propositions of a given form, according to the natural numbers taken as values by the parameters. In other words, we have predicates, for which these parameters are the independent variables. Generally, in a theory, a number of predicates are dealt with simultaneously; but for our investigations it will suffice to consider a theory with respect to some one predicate without reference to other predicates which might be present. Usually, we shall write a one-variable predicate $P(a)$, though the discussion applies equally well to a predicate $P\left(a_{1}, \cdots, a_{n}\right)$ of $n$ variables.

12. Algorithmic theories. As one choice of the objective, we can ask that the theory should give us an effective means for deciding, for any given one of the propositions which are taken as values of the predicate, whether that proposition is true or false. Examples of predicates for which a theoretical conquest of this kind has been obtained are: $a$ is divisible by $b$ (that is, in symbols, $(E x)[a=b x]), a x+b y=c$ is solvable for $x$ and $y$ (that is, $(E x)(E y)[a x+b y=c])$. We shall call this kind of theory for a predicate a complete algorithmic theory for the predicate.

Let us examine the notion of this kind of theory more closely. In setting up a complete algorithmic theory, what we do is to describe a procedure, performable for each set of values of the independent variables, which procedure necessarily terminates and in such manner that from the outcome we can read a definite answer, "Yes" or "No," to the question, "Is the predicate value true?"

We can express this by saying that we set up a second predicate: the procedure terminates in such a way as to give the affirmative answer. The second predicate has the same independent variables as the first, is equivalent to the first, and the determinability of the truth or falsity of its values is guaranteed. 
This last property of the second predicate we designate as the property of being effectively decidable.

Of course the original predicate becomes effectively decidable, in a derivative sense, as soon as we have its equivalence to the second; extensionally, the two are the same. But while our terminology is ordinarily extensional, at this point the essential matter can be emphasized by using the intensional language. The reader may if he wishes write in more explicit statements referring to the (generally) differing objects or processes with which the two predicates are concerned.

Now, the recognition that we are dealing with a well defined process which for each set of values of the independent variables surely terminates so as to afford a definite answer, "Yes" or "No," to a certain question about the manner of termination, in other words, the recognition of effective decidability in a predicate, is a subjective affair. Likewise, the recognition of what may be called effective calculability in a function. We may assume, to begin with, an intuitive ability to recognize various individual instances of these notions. In particular, we do recognize the general recursive functions as being effectively calculable, and hence recognize the general recursive predicates as being effectively decidable.

Conversely, as a heuristic principle, such functions (predicates) as have been recognized as being effectively calculable (effectively decidable), and for which the question has been investigated, have turned out always to be general recursive, or, in the intensional language, equivalent to general recursive functions (general recursive predicates). This heuristic fact, as well as certain reflections on the nature of symbolic algorithmic processes, led Church to state the following thesis $\left({ }^{22}\right)$. The same thesis is implicit in Turing's description of computing machines $\left({ }^{23}\right)$.

Thesis I. Every effectively calculable function (effectively decidable predicate) is general recursive.

Since a precise mathematical definition of the term effectively calculable (effectively decidable) has been wanting, we can take this thesis, together with the principle already accepted to which it is converse, as a definition of it for the purpose of developing a mathematical theory about the term. To the extent that we have already an intuitive notion of effective calculability (effective decidability), the thesis has the character of an hypothesis-a point emphasized by Post and by Church $\left({ }^{24}\right)$. If we consider the thesis and its converse as definition, then the hypothesis is an hypothesis about the application of the mathematical theory developed from the definition. For the acceptance of the hypothesis, there are, as we have suggested, quite compelling grounds.

(22) Church [1].

(23) Turing [1].

(24) Post [1, p. 105], and Church [2]. 
A full account of these is outside the scope of the present paper $\left({ }^{25}\right)$. We are here concerned rather to present the consequences.

In the intensional language, to give a complete algorithmic theory for a predicate $P(a)$ now means to find an equivalent effectively decidable predicate $Q(a)$. It would suffice that $Q(a)$ be given as a general recursive predicate; and by Thesis I, if $Q(a)$ is not so given, then at least there is a general recursive predicate $R(a)$ equivalent to $Q(a)$ and hence to $P(a)$. Thus to give a complete algorithmic theory for $P(a)$ means to find an equivalent general recursive predicate $R(a)$, or more briefly, to express $P(a)$ in the form $R(a)$ where $R$ is general recursive. This predicate form is the one listed first in Theorem II ; and Theorem II gives to each of the other forms a predicate not expressible in that form. Thus, while under our interpretations there is a complete algorithmic theory for each predicate of the form $R(a)$ where $R$ is general recursive, to each of the other forms there is a predicate for which no such theory is possible. We state this in the following theorem, using the particular examples for the one-quantifier forms which were exhibited in the proof of Theorem II.

THEOREM VII. There exists no complete algorithmic theory for either of the predicates $(E x) T_{1}(a, a, x)$ and $(x) \bar{T}_{1}(a, a, x)$.

Of course, once the definition of effective decidability is granted, which affords an enumeration of the effectively decidable predicates, Cantor's methods immediately give other predicates. This theorem, as additional content, shows the elementary forms which suffice to express such predicates.

Abstracting from the particular examples used here, the theorem is Church's theorem on the existence of an unsolvable problem of elementary number theory, and the corresponding theorem of Turing in terms of his machine concept $\left({ }^{26}\right)$. The unsolvability is in the sense that the construction called for by the problem formulation, which amounts to that of a recursive $R$ with a certain property, is impossible. The theorem itself constitutes solution in a negative sense.

13. Formal deductive theories. A second possibility for giving theoretic cohesion to the totality of true propositions taken as values of a predicate $P(a)$ is that offered by the postulational or deductive method. We should like all and only those of the predicate values which are true to be deducible from given axioms by given rules of inference. To make the axioms and principles of inference quite explicit, according to modern standards of rigor, we shall suppose them constituted into a formal system (symbolic logic), in which the propositions taken as values of the predicate are expressible. Those and only those of the formulas expressing the true instances of the predicate

\footnotetext{
${ }^{(25)}$ For a resume, see Kleene [4, Footnote 2], where further references are given.

(26) Turing $[1, \S 8]$.
} 
should be provable. We call this kind of theory for a predicate $P(a)$ a complete formal deductive theory for the predicate.

This type of theory should of course not be confused with incompletely formalized axiomatic theories, such as the theory of natural numbers itself as based on Peano's axioms.

It is convenient in discussing a formal system to name collectively as the "postulates" the rules describing the formal axioms and the rules of inference.

Let us now examine more closely the concept of provability in a stated formal system. If the formalization does accomplish its purpose of making matters explicit, we should be able effectively to recognize each step of a formal proof as an application of a postulate of the system. Furthermore, if the system is to constitute a theory for the predicate $P(a)$, we should be able effectively to recognize, to each natural number $a$, a certain formula of the system which is taken as expressing the proposition $P(a)$. Together, these conditions imply that we should be able, given any sequence of formulas which might be submitted as a proof of $P(a)$ for a given $a$, to check it, thus determining effectively whether it is actually such or not.

Let us introduce a designation for the metamathematical predicate with which we deal in making this check, for a given formal system and predicate $P(a)$.

$\Re(a, \mathrm{X}): \mathrm{X}$ is a proof in the formal system of the formula expressing the proposition $P(a)$.

Then the concept of provability in the system of the formula expressing $P(a)$, or briefly, the provability of $P(a)$, is expressible as $(E \mathrm{X}) \Re(a, \mathrm{X})$.

As we have just argued, the predicate $\Re(a, \mathrm{X})$ should be an effectively decidable metamathematical predicate. Here the formal objects over which $\mathrm{X}$ ranges, if the notation of the system is explicit, should be given in some manner which affords an effective enumeration of them. Using the indices in this enumeration, or generally any effective Gödel numbering of the formal objects, the metamathematical predicate $\Re(a, \mathrm{X})$ will be carried into a numbertheoretic predicate $R(a, x)$, taken as false for any $x$ not correlated to a formal object, which should then also be effectively decidable. By Thesis I, the effective decidability of the latter implies its general recursiveness. We are thus led to state a second thesis.

THESIs II. For any given formal system and given predicate $P(a)$, the predicate that $P(a)$ is provable is expressible in the form $(E x) R(a, x)$ where $R$ is general recursive.

This thesis corresponds to the standpoint that the role of a formal deductive system for a predicate $P(a)$ is that of making explicit the notion of what constitutes a proof of $P(a)$ for a given $a$. If a proposed "formal system" for $P(a)$ does not do this, we should say that it is not a formal system in the 
strict sense, or at least not one for $P(a)$. Taken this way, the thesis has a definitional character.

Presupposing, on the other hand, a prior conception of what constitutes a formal system for a given predicate in the strict sense, the thesis has the character of an hypothesis, to which we are led both heuristically and from Thesis I by general considerations.

Conversely, if a predicate of the form $(E x) R(a, x)$ where $R$ is general recursive is given, it is easily seen that we can always set up a formal system of the usual sort, with an explicit criterion of proof, in which all true instances of this predicate and only those are provable.

Using the thesis, and this converse, we can now say that to give a complete formal deductive theory for a predicate $P(a)$ means to find an equivalent predicate of the form $(E x) R(a, x)$ where $R$ is general recursive, or more briefly, to express the predicate in this form. By Theorem II, there are predicates of the other one-quantifier form, and of the forms with more quantifiers, not expressible in this form. Hence while there are complete formal deductive theories to each predicate of either of the forms $R(a)$ and $(E x) R(a, x)$ where $R$ is general recursive, to each of the other forms there is a predicate for which no such theory is possible. Specifically, using the one-quantifier example given in the proof of Theorem II :

THEOREM VIII. There is no complete formal deductive theory for the predicate $(x) \bar{T}_{1}(a, a, x)$.

This is the famous theorem of Gödel on formally undecidable propositions, in a generalized form. A proposition is formally undecidable in a given formal system if neither the formula expressing the proposition nor the formula expressing its negation is provable in the system. Gödel gave such a proposition for a certain formal system (by a method evidently applying to similar systems), subject to the assumptions of the consistency and $\omega$-consistency of the system. Later Rosser gave another proposition, for which the latter assumption is dispensed with $\left({ }^{27}\right)$.

In the present form of the theorem, we have a preassigned predicate $(x) \bar{T}_{1}(a, a, x)$ and a method which, to any formal system whatsoever for this predicate, gives a number $f$ for which the following is the situation.

Suppose that the system meets the condition that the formula expressing the proposition $(x) \bar{T}_{1}(f, f, x)$ is provable only if that proposition is true. Then the proposition is true but the formula expressing it unprovable. This statement of results uses the interpretation of the formula, but if the system has certain ordinary deductive properties for the universal quantifier and recursive predicates, our condition on the system is guaranteed by the metamathematical one of consistency.

If the system contains also a formula expressing the negation of

(27) Rosser [1]. 
$(x) \bar{T}_{1}(f, f, x)$, and if the system meets the further condition that this formula is provable only if true, then this formula cannot be provable, and we have a formally undecidable proposition. The further condition, if the system has ordinary deductive properties, is guaranteed by the metamathematical one of $\omega$-consistency.

Moreover, we can incorporate Rosser's elimination of the hypothesis of $\omega$-consistency into the present treatment. To do so, we replace the predicate $(E x) R(a, x)$ for the application of Theorem II by $(E x)[R(a, x) \&(y)[y<x$ $\rightarrow \bar{S}(a, y)]$ ] where $(E x) S(a, y)$ is the predicate expressing the provability of the negation of $(x) \bar{T}_{1}(a, a, x)$. This changes the $f$ for the system.

Thus we come out with the usual metamathematical results for a given formal system.

For the case that a formal system is sought which should not only prove the true instances of $P(a)$ but also refute the false ones, if the classical law of the excluded middle is applied to the propositions $P(a)$, then the Gödel theorem (Theorem VIII) comes under the Church theorem (Theorem VII). For had we completeness with respect both to $P(a)$ and to $\bar{P}(a)$, we could obtain a general recursive $R(a)$ equivalent to the given predicate by the method used in proving the second part of Theorem V. Informally, this amounts merely to the remark that we should have the algorithm for $P(a)$ which consists in searching through some list of the provable formulas until we encounter either the formula expressing $P(a)$ or the formula expressing $\bar{P}(a)$.

The connection between Gödel's theorem and the paradoxes has been much noted. The author gave a proof of Gödel's theorem along much the present lines but as a refinement of the Richard paradox rather than of the Epimenides $\left({ }^{28}\right)$. That gave the undecidable propositions as values of a predicate of the more complicated form $(x)(E y) R(a, x, y)$ where $R$ is general recursive. The Epimenides paradox now appears as the more basic. Currently, Curry has noted the same phenomenon in connection with the Kleene-Rosser inconsistency theorem $\left({ }^{29}\right)$.

14. Discussion, incomplete theories. In the present form of Gödel's theorem, several aspects are brought into the foreground which perhaps were not as clearly apparent in the original version.

Not merely, to any given formal system of the type considered, can a proposition be formulated with respect to which that system is incomplete, but all these propositions can be taken as values of a preassignable elementary predicate, with respect to which predicate therefore no system can be complete. This depends on the thesis giving a preassignable form to the concept of provability in a formal system.

(28) Kleene [2, XIII].

(29) Kleene and Rosser [1], Curry [2]. 
For the interpretation of the propositions we have required, as minimum, only the notions of effectively calculable predicates and of the quantifiers used constructively. It seems that lesser presuppositions, if one is to allow any mathematical infinite, are hardly conceivable.

Beyond that the system should fulfil the structural characteristic expressed in Thesis II, and should yield results correct under this modicum of interpretation, we have need of no reference whatsoever to its detailed constitution.

In particular, the nature of the intuitive evidence for the deductive processes which are formalized in the system plays no role.

Let us imagine an omniscient number theorist, whom we should expect, through his ability to see infinitely many facts at once, to be able to frame much stronger systems than any we could devise. Any correct system which he could reveal to us, telling us how it works without telling us why, would be equally subject to the Gödel incompleteness.

It is impossible to confine the intuitive mathematics of elementary propositions about integers to the extent that all the true theorems will follow from explicitly stated axioms by explicitly stated rules of inference, simply because the complexity of the predicates soon exceeds the limited form representing the concept of provability in a stated formal system.

We selected as the objective in constructing a formal deductive system that what constitutes proof should be made explicit in the sense that a proposed proof could be effectively checked, and either declared formally correct or declared formally incorrect.

Let us for the moment entertain a weaker conception of a formal system, under which, if we should happen to discover a correct proof of a proposition or be presented with one, then we could check it and recognize its formal correctness, but if we should have before us an alleged proof which is not correct, then we might not be able definitely to locate the formal fallacy. In other words, under this conception a system possesses a process for checking, which terminates in the affirmative case, but need not in the negative. Then the concept of provability would have the form $(E x) P^{+}(a, x)$ where $P^{+}$is the positive completion of a partial recursive predicate $P(a, x)$. By Theorem VI, $P^{+}(a, x)$ is expressible in the form $(E y) R(a, x, y)$ where $R$ is general recursive. Then the provability concept has the form $(E x)(E y) R(a, x, y)$, or by contraction of quantifiers $(E x) R\left(a,(x)_{1},(x)_{2}\right)$. This is of the form $(E x) R(a, x)$ where $R$ is general recursive. Thus the concept of provability has the usual form, and Gödel's theorem applies as before. If we take a new concept of proof based on $R(a, x)$, that is, if we redesignate the steps in the checking process as the formal proof steps, the concept of proof assumes the usual form.

We gave no attention, when we formulated the objectives both of an algorithmic and of a formal deductive theory, to the nature of the evidence for the correctness of the theory, or to various other practical considerations, 
simply because the crude structural objectives suffice to entail the corresponding incompleteness theorems. In this connection, it may be of some interest to give the corresponding definitions, although these may not take into account all the desiderata, for the case of incomplete theories of the two sorts. We shall state these for predicates of $n$ variables $a_{1}, \cdots, a_{n}$, as we could also have done for the case of the complete theories.

To give an algorithmic theory (not necessarily complete) for a predicate $P\left(a_{1}, \cdots, a_{n}\right)$ is to give a general recursive function $\pi\left(a_{1}, \cdots, a_{n}\right)$, taking only 0,1 , and 2 as values, such that

$$
\left\{\begin{array}{l}
\pi\left(a_{1}, \cdots, a_{n}\right)=0 \rightarrow P\left(a_{1}, \cdots, a_{n}\right) \\
\pi\left(a_{1}, \cdots, a_{n}\right)=1 \rightarrow \bar{P}\left(a_{1}, \cdots, a_{n}\right) .
\end{array}\right.
$$

The algorithm always terminates, but if $\pi\left(a_{1}, \cdots, a_{n}\right)$ has the value 2 we can draw no conclusion about $P\left(a_{1}, \cdots, a_{n}\right)$.

To give a formal deductive theory (not necessarily complete) for a predicate $P\left(a_{1}, \cdots, a_{n}\right)$ is to give a general recursive predicate $R\left(a_{1}, \cdots, a_{n}, x\right)$ such that

$$
(E x) R\left(a_{1}, \cdots, a_{n}, x\right) \rightarrow P\left(a_{1}, \cdots, a_{n}\right) .
$$

In words, to give a formal deductive theory for a predicate $P\left(a_{1}, \cdots, a_{n}\right)$ is to find a sufficient condition for it of the form $(E x) R\left(a_{1}, \cdots, a_{n}, x\right)$ where $R$ is general recursive. Here, according to circumstances, the sufficiency may be established from a wider context, or it may be a matter of postulation (hypothesis), or of conviction (belief).

From the present standpoint, the setting up of this sufficient condition is the essential accomplishment in the establishment of a so-called metatheory (in the constructive sense) for the body of propositions taken as the values of a predicate. We note that this may be accomplished without necessarily going through the process of setting up a formal object language, from which $R$ is obtainable by subsequent arithmetization, although as remarked above, we can always set up the object language, if we have the $R$ by some other means.

In the view of the present writer, the interesting variations of formal technique recently considered by Curry have the above as their common feature with formalization of the more usual sort $\left({ }^{30}\right)$. This is stated in our terminology, Curry's use of the terms "meta" and "recursive" being different. He gives examples of "formal systems," in connection with which he introduces some predicates by what he calls "recursive definitions," but what we should prefer to call "inductive definitions." This important type of definition, under suitable precise delimitation so that the individual clauses are constructive, can be shown to lead always to predicates expressible in the form $(E x) R\left(a_{1}, \cdots, a_{n}, x\right)$ where $R$ is recursive in our sense. Indeed, this fact
(30) Curry [1]. 
can be recognized by substantially the method indicated above for the case of the inductive definition establishing the notion of provability for a formal system of the usual sort.

Conversely, given any predicate expressible in the form $(E x) R\left(a_{1}, \cdots\right.$, $\left.a_{n}, x\right)$ where $R$ is recursive, we can set up an inductive definition for it.

15. Ordinal logics. In ordinal logics, studied by Turing $\left({ }^{31}\right)$, the requirement of effectiveness for the steps of deduction is relaxed to allow dependence on a number (or $\lambda$-formula) which represents an ordinal in the ChurchKleene theory of constructive ordinals $\left({ }^{32}\right)$. A presumptive proof in an ordinal logic cannot in general be checked objectively, since the proof character depends on the number which occupies the role of a Church-Kleene representative of an ordinal actually being such, for which there is no effective criterion. Nevertheless it was hoped that ordinal logics could be used to give complete orderings (with repetitions) of the true propositions of certain forms into transfinite series, by means of the ordinals represented in the proofs, in such a way that the proving of a proposition in the ordinal logic (and therewith the determination of a position for it in the series) would somehow make it easier to recognize the truth of the proposition.

Turing obtained a number of interesting results, largely outside the scope of this article, but among them the following. There are ordinal logics which are complete for the theory of a predicate of the form $(x)(E y) R(a, x, y)$ where $R$ is general recursive; however, for the example of such a logic which is given, its use would afford no theoretic gain, since the recognition that the number which plays the role of ordinal representative in a proof of the logic is actually such comes to the same as the direct recognition of the truth of the proposition proved.

Now let us approach the topic by inquiring whether, and if so where, the property of being provable in a given ordinal logic is located in the scale of predicate forms of Theorem II. First, it turns out that the property of a number $a$ of being the representative of an ordinal is expressible in the form $(x)(E y) R(a, x, y)$ where $R$ is recursive $\left({ }^{33}\right)$. Now we may use the definition of ordinal logic in terms of $\lambda$-conversion, or we may take the notion in general terms as described above, and state the thesis that for a given predicate $P(a)$ and given ordinal logic the provability of $P(a)$ is expressible in the form $(E \alpha)(E x) R(a, \alpha, x)$ where $\alpha$ ranges over the ordinal representatives and $R$ is general recursive. In either case, it then follows that the provability of $P(a)$ is expressible in the form $(E x)(y)(E z) R(a, x, y, z)$ where $R$ is general recursive. Conversely, to any predicate of the latter form, we can find an ordinal logic

(31) Turing [2]. Turing gave a somewhat restricted definition of "ordinal logic" in terms of the theory of $\lambda$-conversion for predicates expressible in the form $(x)(E y) R(a, x, y)$ where $R$ is recursive.

(2z) Church and Kleene [1], Church [2], Kleene [4].

(33) Kleene [5]. 
in the more general sense such that provability in the logic expresses the predicate. Hence there is a complete ordinal logic to each predicate of each of the forms

$$
\begin{array}{rlll}
R(a) & (E x) R(a, x) & (x)(E y) R(a, x, y) & (E x)(y)(E z) R(a, x, y, z) \\
(x) R(a, x) & (E x)(y) R(a, x, y)
\end{array}
$$

where $R$ is general recursive, but by Theorem II, classically there are predicates of the form $(x)(E y)(z) R(a, x, y, z)$ and of each of the forms with more quantifiers, or classically and intuitionistically of the form $(\overline{E x})(y)(E z) R(a, x$, $y, z)$ and of the negation of each of the forms with more quantifiers, for which no complete ordinal logic is possible. Specifically:

THEOREM IX. There is no complete ordinal logic for the predicate $(\overline{E x})(y)(E z) T_{3}(a, a, x, y, z)$.

Ordinal logics form a class of examples of the systems of propositions which have recently come under discussion, in which more or less is retained of the ordering of propositions in deductive reasoning, but with an extension into the transfinite, or a sacrifice of constructiveness in individual steps. These may be called "non-constructive logics," in contrast to the formal deductive systems in the sense of $\$ \$ 13-14$ which are "constructive logics." In general, the usefulness of a non-constructive logic may be considered to depend on the degree to which the statement of the non-constructive proof criterion is removed from the direct statement of the propositions.

Theorem IX is a "Gödel theorem" for the ordinal logics. The ordinal logics were at least conceived with somewhat of a constructive bias. Rosser has shown how Gödel theorems arise on going very far in the direction of nonconstructiveness $\left({ }^{34}\right)$, and Tarski has stated the Gödel argument for systems of sentences in general $\left.{ }^{35}\right)$. Incidental of Rosser's results for finite numbers of applications of the Hilbert "rule of infinite induction," also called "Carnap's rule," can easily be inferred from Theorem II, through the obvious correspondence of an application of this rule to a universal quantifier in the proof concept. However, the proof concepts for non-constructive logics soon outrun the scale of predicate forms of Theorem II. This appears to be the case even for the extension to protosyntactical definability given by Quine $\left({ }^{36}\right)$. If one is going very far in the direction of non-constructiveness, and is not interested in considerations of the sort emphasized in $\$ \$ 12-14$, there is no advantage in starting from the theory of recursive functions. But the more general results do not detract from the special significance which attaches to the Gödel theorems associated with provability criteria of the forms $R(a)$ and $(E x) R(a, x)$
(34) Rosser [2].
(35) Tarski [2].
(36) Quine [1]. 
where $R$ is general recursive, that is, Church's theorem and Gödel's theorem, for which forms only it is true that a given proof is a finite object.

16. Constructive existence proofs. A proof of an existential proposition $(E y) A(y)$ is acceptable to an intuitionist, only if in the course of the proof there is given a $y$ such that $A(y)$ holds, or at least a method by which such a $y$ could be constructed. Consider the case that $A(y)$ depends on other variables. Say that there is one of these, $x$, and rewrite the proposition as $(x)(E y) A(x, y)$. The proposition asserts the existence of a $y$ to each of the infinitely many values of $x$. In this case, the only way in which the constructivist demand could in general be met would be by giving the $y$ as an effectively calculable function of $x$, that is, by giving the function. According to Thesis $I$, this function would have to be general recursive. Hence we propose the following thesis (and likewise for $n$ variables $x_{1}, \cdots, x_{n}$ ):

ThesIs III. A proposition of the form $(x)(E y) A(x, y)$ containing no free variables is provable constructively, only if there is a general recursive function $\phi(x)$ such that $(x) A(x, \phi(x))$.

When such a $\phi$ exists, we shall say that $(x)(E y) A(x, y)$ is recursively fulfillable $\left({ }^{37}\right)$.

This thesis expresses what seems to be demanded from the standpoint of the intuitionists. Whether such explicit rules of proof as they have stated do conform to the thesis is a further question which will be considered elsewhere $\left({ }^{38}\right)$. However, in its aspect as restriction on all intuitionistic existence proofs, the possibilities for which, as we know by Theorem VIII, transcend the limitations of any preassignable formal system, the thesis is more general than a metamathematical result concerning a given system.

We now examine the notion of recursive fulfillability as it applies to the values of a given predicate of the form $(x)(E y)(z) R(a, x, y, z)$ where $R$ is general recursive. Select any fixed value of $a$. Given a recursive $\phi$ which fulfils the corresponding proposition, by Theorem IV there is a number $e$ such that $(x)(E y) T_{1}(e, x, y)$ and $(x)(y)\left[T_{1}(e, x, y) \rightarrow(z) R(a, x, U(y), z)\right]$. Conversely, if such an $e$ exists, the proposition is fulfilled by the general recursive function $U\left(\mu y T_{1}(e, x, y)\right)$. Thus

$$
(E e)\left\{(x)(E y) T_{1}(e, x, y) \&(x)(y)\left[T_{1}(e, x, y) \rightarrow(z) R(a, x, U(y), z)\right]\right\}
$$

is a necessary and sufficient condition for recursive fulfillability. When the quantifiers are suitably brought to the front and contracted, this assumes the form $(E x)(y)(E z) R(a, x, y, z)$ with another general recursive $R$ depending on the original $R$.

By Theorem II, classically, there is a predicate of the original form

(37) A further analysis of the implications of constructive provability is given in Kleene [6].

(38) Nelson [1]. 
$(x)(E y)(z) R(a, x, y, z)$ which is not expressible in this form $(E x)(y)(E z) R(a, x, y, z)$, in which the condition of its recursive fulfillability is expressible.

Using the example of such a predicate given in the proof of Theorem II, we have then

$$
\left\{(x)(E y)(z) \bar{T}_{3}(a, a, x, y, z) \text { rec. fulf. }\right\} \equiv(E x)(y)(E z) R(a, x, y, z)
$$

for a certain general recursive $R$. Substituting the number $f$ of (14) for $a$ in (14) and (38),

$$
\begin{aligned}
(E x)(y)(E z) R(f, x, y, z) & \equiv(E x)(y)(E z) T_{3}(f, f, x, y, z), \\
\left\{(x)(E y)(z) \bar{T}_{3}(f, f, x, y, z) \text { rec. fulf. }\right\} & \equiv(E x)(y)(E z) R(f, x, y, z) .
\end{aligned}
$$

By the definition of recursive fulfillability,

$$
\left\{(x)(E y)(z) \bar{T}_{3}(f, f, x, y, z) \text { rec. fulf. }\right\} \rightarrow(x)(E y)(z) \bar{T}_{3}(f, f, x, y, z) .
$$

Suppose that $(x)(E y)(z) \bar{T}_{3}(f, f, x, y, z)$ were recursively fulfillable. We could then conclude by (40) and (39), $(E x)(y)(E z) T_{3}(f, f, x, y, z)$, and by (41), $(x)(E y)(z) \bar{T}_{3}(f, f, x, y, z)$. These results are incompatible. Therefore by reductio ad absurdum, $(x)(E y)(z) \bar{T}_{3}(f, f, x, y, z)$ is not recursively fulfillable, and hence by Thesis III not constructively provable.

Now by (40) and (39), we have $(\overline{E x})(y)(E z) T_{3}(f, f, x, y, z)$; and thence classically we can proceed to $(x)(E y)(z) \bar{T}_{3}(f, f, x, y, z)$.

Theorem X. For a certain number $f$, the proposition $(x)(E y)(z) \bar{T}_{3}(f, f, x, y, z)$ is true classically, but not constructively provable.

Notice that we have here a fixed unprovable proposition for all constructive methods of reasoning, whereas in the preceding incompleteness theorems. we had only an infinite class of propositions, some of which must be unprovable in a given theory.

Intuitionistic number theory has been presented as a subsystem of the classical, so that the intuitionistic results hold classically, though many classical results are not asserted intuitionistically. The possibility now appears of extending intuitionistic number theory by incorporating Thesis III in the form

$$
(x)(E y) A(x, y) \rightarrow\{\text { for some general recursive } \phi,(x) A(x, \phi(x))\},
$$

so that the two number theories should diverge, with the proposition of Theorem $\mathrm{X}$ true classically, and its negation true intuitionistically $\left({ }^{(29)}\right.$.

For the classical proof, an application of

$$
\overline{(x)} A(x) \rightarrow(E x) \bar{A}(x)
$$

suffices as the sole non-intuitionistic step; therewith that law of logic would

(39) This is perhaps hinted in Church [1, first half of p. 363]. 
be refuted intuitionistically, for a certain $A$. Hitherto the intuitionistic refutations of laws of the classical predicate calculus have depended on the interpretation of the quantifiers in intuitionistic set theory $\left({ }^{40}\right)$.

The result of Theorem $\mathrm{X}$, with another proposition as example, can be reached as follows. Consider the proposition,

$$
(x)(E y)\left\{\left[(E z) T_{1}(x, x, z) \& y=0\right] \vee\left[(z) \bar{T}_{1}(x, x, z) \& y=1\right]\right\} .
$$

This holds classically, by application of the law of the excluded middle in the form

$$
(x)\{(E z) A(x, z) \vee(z) \bar{A}(x, z)\} \text {, }
$$

or the form

$$
(x)(A(x) \vee \bar{A}(x))
$$

from which the other follows by substituting $(E z) A(x, z)$ for $A(x)$. But it is not recursively fulfillable, since it can be fulfilled only by the representing function of the predicate $(E z) T_{1}(x, x, z)$, which, as we saw in the proof of Theorem II, is non-recursive.

17. Non-elementary predicates. The elementary predicates are enumerable. By Cantor's methods, there are therefore non-elementary number-theoretic predicates. However let us ask what form of definition would suffice to give such a predicate. Under classical interpretations, the enumeration of predicate forms given in Theorem II for $n$ variables suffices for the expression of every elementary predicate of $n$ variables. By defining relations of the form shown in the next theorem, we can introduce a predicate $M(a, k)$ so that it depends for different values of $k$ on different numbers of alternating quantifiers. On the basis of Theorem II, it is possible to do this in such a way that the predicate will be expressible in none of the forms of Theorem II.

Theorem XI. Classically, there is a non-elementary predicate $M(a, k) d e$ finable by relations of the form

$$
\left\{\begin{aligned}
M(a, 0) & \equiv R(a) \\
M(a, 2 k+1) & \equiv(E x) M(\phi(a, x), 2 k) \\
M(a, 2 k+2) & \equiv(x) M(\phi(a, x), 2 k+1)
\end{aligned}\right.
$$

where $R$ and $\phi$ are primitive recursive.

We are dealing here with essentially the same fact which Hilbert-Bernays discover by setting up a truth definition for their formal system $(Z)\left({ }^{41}\right)$.

The system $(Z)$ has as primitive terms only ', $+, \cdot,=$ and the logical operations. The predicates expressible in these terms are elementary. Con-

(40) Heyting [1, p. 65].

(11) Hilbert and Bernays [1, pp. 328-340]. 
versely, using Theorem IV and Gödel's reduction of primitive recursive functions to these terms $\left({ }^{42}\right)$, every elementary predicate is expressible in $(Z)$.

The Hilbert-Bernays result is an application to $(Z)$ of Tarski's theorem on the truth concept $\left({ }^{40}\right)$, with the determination of a particular form of relations which give the truth definition for $(Z)$. If $(Z)$ is consistent, a formal proof that the relations do define a predicate is beyond the resources of $(Z)$.

\section{BIBLIOGRAPHY}

Alonzo Church

1. An unsolvable problem of elementary number theory, Amer. J. Math. vol. 58 (1936) pp. 345-363.

2. The constructive second number class, Bull. Amer. Math. Soc. vol. 44 (1938) pp. 224-232. Alonzo Church and S. C. Kleene

1. Formal definitions in the theory of ordinal numbers, Fund. Math. vol. 28 (1936) pp. 11-21. Alonzo Church and BarkLey Rosser

1. Some properties of conversion, Trans. Amer. Math. Soc. vol. 39 (1936) pp. 472-482.

H. B. CURRY

1. Some aspects of the problem of mathematical rigor, Bull. Amer. Math. Soc. vol. 47 (1941) pp. 221-241.

2. The inconsistency of certain formal logics, J. Symbolic Logic vol. 7 (1942) pp. 115-117. KURT GÖDEL

1. Über formal unentscheidbare Sätze der Principia Mathematica und verwandter Systeme I, Monatshefte für Mathematik und Physik vol. 38 (1931) pp. 173-198.

2. On undecidable propositions of formal mathematical systems, notes of lectures at the Institute for Advanced Study, 1934.

David Hilbert and Paul Bernays

1. Grundlagen der Mathematik, vol. 2, Berlin, Springer, 1939.

Arend Heyting

1. Die formalen Regeln der intuitionistischen Mathematik, Preuss. Akad. Wiss. Sitzungsber, Phys.-math. Kl. 1930, pp. 57-71, 158-169.

S. C. KLEENE

1. A theory of positive integers in formal logic, Amer. J. Math. vol. 57 (1935) pp. 153-173, 219-244.

2. General recursive functions of natural numbers, Math. Ann. vol. 112 (1936) pp. 727-742.

3. A note on recursive functions, Bull. Amer. Math. Soc. vol. 42 (1936) pp. 544-546.

4. On notation for ordinal numbers, J. Symbolic Logic vol. 3 (1938) pp. 150-155.

5. On the forms of the predicates in the theory of constructive ordinals, to appear in Amer. J. Math. (Bull. Amer. Math. Soc. abstract 48-5-215).

6. On the interpretation of intuitionistic number theory, Bull. Amer. Math. Soc. abstract 48-1-85.

S. C. KLEene aNd Barkley Rosser

1. The inconsistency of certain formal logics, Ann. of Math. (2) vol. 36 (1935) pp. 630-636. David Nelson

1. Recursive functions and intuitionistic number theory, under preparation.

E. L. Post

1. Finite combinatory processes-formulation I, J. Symbolic Logic vol. 1 (1936) pp. 103-105.

(42) Gödel [1, Theorem VII]. See Kleene [3 (erratum: p. 544, line 11, "of" should be at the end of the line)].

(43) Tarski [1]. 
W. V. QUINE

1. Mathematical logic, New York, Norton, 1940.

BARKLEY ROSSER

1. Extensions of some theorems of Gödel and Church, J. Symbolic Logic vol. 1 (1936) pp. 8791.

2. Gödel theorems for non-constructive logics, ibid. vol. 2 (1937) pp. 129-137.

ALFRED TARSKI

1. Der Wahrheitsbegriff in den formalisierten Sprachen, Studia Philosophica vol. 1 (1936) pp. 261-405. (Original in Polish, 1933.)

2. On undecidable statements in enlarged systems of logic and the concept of truth, J. Symbolic Logic vol. 4 (1939) pp. 105-112.

A. M. TURING

1. On computable numbers, with an application to the Entscheidungsproblem, Proc. London Math. Soc. (2) vol. 42 (1937) pp. 230-265.

2. Systems of logic based on ordinals, ibid. vol. 45 (1939) pp. 161-228.

Amherst College,

Amherst, Mass. 